\title{
Influence mechanism of congestion degree of layout on domino effect in petrochemical plants
}

\author{
Huang Wenduo ${ }^{1}$, Shao Xuechun ${ }^{1}$, Wang Jiajie ${ }^{1}$, Xiao Yang $^{1}$,Sun Dongliang ${ }^{1 *}$ \\ ${ }^{1}$ State Environmental Protection Key Laboratory of Environmental Risk Assessment and Control on Chemical Process, School of \\ Resources and Environmental Engineering, East China University of Science and Technology, Shanghai 200237, China
}

\begin{abstract}
The study focused on the establishment of the congestion degree in describing the layout of process plants, and discovering the influence mechanism of congestion degree on the risk of domino effect, in view of the fact that the congestion degree has not been used to represent the characteristics of petrochemical plant layout. A characterization model was built for the congestion degree of process plant layouts applying the theory of fuzzy model that it took safety distance, the amount of materials involved and the hazard of these substances into consideration, and came out with a membership function through logical calculus, which made congestion degree a comparable data. Secondly, a systematic domino effect risk analysis method was generalized on account of domino effect quantitative risk analysis method which include the equipment damage probability, the domino effect occurrence probability, risk analysis based on the consequences, individual risk index and social risk index, the process plant natural risk index and real risk index, and then these indices were compared to select the most suitable ones to illustrate the risk of domino effect and to research about part of the storage tank field of a petrochemical plant. Finally, the influence mechanism of layout congestion degree on domino effect of regional accident was obtained through this quantitative analysis. Simultaneously, a range of the congestion degree was acquired according to the individual risk index which can be used as a reference in the design stage of the process plant. The result proved that high congestion degree in process plant greatly increased the risk of domino effect in most of the quantitative risk analysis index, such as the probability of resulting in secondary accidents, the harm on both human and the equipments caused by the initial accident, and the individual risk and social risk, etc. The characterization models for the congestion degree and the domino risk analysis method established and summarized in the study have been applied in the design stage of process plant layout to optimize the layout, and thus reduce the risk of domino effect caused by accidents.
\end{abstract}

\section{Introduction}

It will inevitably increase the amount of hazardous substances in the unit facilities as the chemical production equipment of modern industry is gradually developing towards large-scale, complex and intensive, which will greatly raise the probability of domino accidents and the severity of their consequences. As early as 1996, the Seveso Act was promulgated in Europe as an important reference standard for the study of domino accidents (Alileche N., Cozzani V., \& Reniers G., 2015). In 1976, the concept of domino effect was defined for the first time in a series of major hazard control reports published by the Advisory Committee on Major Hazards of the HSC (British Health and Safety Council). In 1998, Khan and Abasi studied the mechanism and expansion mode of triggering domino accidents, and initially established the extended probability model of triggering domino accidents by thermal radiation, overpressure and debris (Khan, F.I. \& Abbasi, S.A., 1998). In 2001, Khan and Abbas (Khan, F.I. \& Abbasi,
S.A., 2001) identified two main modes of escalation of domino effects caused by initial accidents: direct escalation and indirect escalation. In 2004, Valerio Cozzani and Ernesto Salzano (Cozzani and Salzano, 2004) classified the target vessel into four categories: atmospheric pressure vessel, pressure vessel, lengthened vessel and small vessel, and proposed the probability model of overpressure expansion for the target vessel and the corresponding threshold. In 2005, Valerio Cozzani (Cozzani, Gubinelli \& Antonioni, 2005) established a more reasonable thermal radiation expansion probability model for the target vessel. In 2006, Ernesto Salzanoa and Valerio Cozzani (Salzanoa and Cozzani, 2006) studied the leakage probability of process equipment failure caused by shock wave overpressure according to different damage intensity. There have been many studies on risk calculation of domino effect, for example, Analytical Method by Cozzani V. et al. (Cozzani, 2014; Antonioni \& Spadoni, 2009), Monte-Carlo Simulation by Abdolhamidzadeh et al. (Abdolhamidzadeh and Abbasi, 2010; Rad and Abdolharnidzadeh, 2014), Event Tree Analysis Method by Bernechea E.J. et al. (Bernechea E.J., 
Antonio Vilchez J. \& Arnaldos J., 2013), Bayesian Network Method by Khakzad N. et al. (Khakzad N., Khan F. \& Amyotte P., 2013, 2014, 2015). Land planning in different countries was generally divided into three methods: safety distance-based, consequence-based and risk-based (Wu Z. Z., 2004). The plant layout problem was first described as a quadratic allocation problem (QAP) which was extended by some scholars to form mixed integer linear programming (MILP) (Jayakumar and Reklaitis, 1996; Guirardello and Swaney, 2005). After introducing safety factors into plant layout model, MILP problem will be transformed into mixed integer non-linear programming (MINLP) problem. Penteado led into the concept of property loss in risk assessment as a factor of consideration for plant layout (Patsiatzis, Knight \& Papageorgiou, 2004). Valerio Cozzani et al. proposed a domino effect evaluation index based on intrinsic safety distance of domino effect, which can be used to analyze the risk of domino effect in a particular layout (Cozzani, Tugnoli \& Salzano, 2009). Congestion degree was first proposed by Stankey in 1973 as a broad concept of field enviroment congestion degree, including garbage, natural resource abuse and deforestation (Stankey, \& Mc Cool, 1989). Now, this concept is widely used in traffic to reflect the degree of busy traffic. However, the concept of "congestion degree" is not defined in the layout of petrochemical plants.

\section{Methods for establishing the characterization model of congestion degree of petrochemical plant distribution}

The concept of the congestion degree is used to indicate the actual road congestion in road traffic, that is, the distribution of the traffic flow. The establishment of the factory layout congestion degree in the petrochemical enterprises can directly reflect the concentration of the equipment and the material in the layout of the plant area, and then study the impact of plant layout congestion degree on accident severity and accident domino risk. The factors that affect the layout of the plant are not single. The distance between the units in the plant, the volume of the medium in the unit and the risk of the medium in the unit all influence the number of the layout crowding. On the basic of the above three factors, a stochastic-fuzzy model was proposed to characterize the congestion degree of petrochemical plant layout, which can be used to study the impact of the congestion degree of plant layout on the risk of accident domino effect.

\subsection{Stochastic-Fuzzy model for congestion degree of petrochemical plant distribution}

The concept of congestion degree was based on three factors such as the spacing between units in the factory area, the quantity of the medium in the unit and the risk of the medium in the unit. Therefore, the congestion degree of petrochemical plant layout was defined as follows.
$\mathrm{CD}($ congest ion degree $)=\mathrm{CD}_{\mathrm{S}} \times \mathrm{CD}_{\mathrm{V}} \times \mathrm{CD}_{\mathrm{H}}$

The process of establishing and applying fuzzy membership function was as follows communications.

Firstly, determine the fuzzy variables to characterize a factor. For example, for the "medium stock" factor, the dimensionless value of the ratio between the actual stock and the critical mass of the medium was taken as the fuzzy variable.

Secondly, the congestion degree for the selected fuzzy variables was divided. For example, the second levels can be classified as "high (H)" and "low (L)"; the third level can be classified as "high (H)", "medium (M)" and "low (L)".

Thirdly, the fuzzy membership function was created. Generally, the methods of establishing membership functions include $\mathrm{F}$ statistical method, binary contrast method, inference method, and so on. The basic forms of membership functions were consisted of rectangular distribution, trapezoid distribution, normal distribution, Cauchy distribution and so on. The membership functions can be any combination of the above in this paper.

Finally, In view of the fuzzy membership function chart, the congestion degree representation value of the membership degree can be read out according to the value of the fuzzy variable. As was shown in Fig.1, the fuzzy variable $\mathrm{X}$ was divided into five grades: low (L), low-medium (L-M), middle (M), medium-high (M-H) and high $(\mathrm{H})$. If the calculated value of the fuzzy variable is $X_{n}$, the congestion degree is "low (L)", the degree of membership is 0.7 , and the congestion degree is "low-medium (L-M)", and the membership degree is 0.3 , than the fuzzy membership degree can be expressed as

$$
C D=\frac{0.7}{L}+\frac{0.3}{L-M}
$$

From the above steps, the fuzzy function can be established, and in the light of the principle of maximum membership degree of the fuzzy model, the formula of congestion degree can be obtained.

$$
C D=\max _{C D_{S} \times C D_{V} \times C D_{H}}\left[\min \left(C D_{S}, C D_{V}, C D_{H}\right)\right]
$$

where: $C D_{S}$ is the fuzzy membership degree of "unit distance"; $C D_{V}$ is the fuzzy membership degree of "medium inventory"; $C D_{H}$ is the fuzzy membership degree of "medium risk"; $C D_{S} \times C D_{V} \times C D_{H}$ is fuzzy logic language.

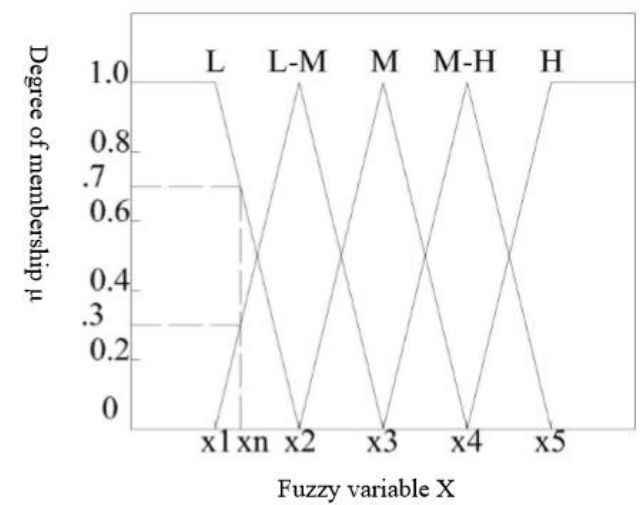

Fig1 Fuzzy membership function diagram 


\subsection{The establishment of the fuzzy membership function of the influencing factors}

\subsubsection{Fuzzy membership function of unit distance}

The fuzzy variable of unit spacing was chosen to express the ratio of the actual spacing $(d)$ between the standard spacing $(D)$ obtained in the specification, expressed as $d / D$. When there are multiple units around the evaluated unit, the group spacing with the maximum value of the fuzzy variable, i.e. the highest degree of congestion, is treated as the unit spacing value. Among them, the standard spacing value comes from "Code for Fire Protection in Design of Petrochemical Enterprises" (GB 50160-2008) and "Code for Fire Protection in Architectural Design" (GB 50016-2014). In this paper, the interval crowding degree was ranked in five grades of "low (L)", low -medium (L-M)", "medium (M)", "medium-high (M-H)" and "high (H)", which can get the fuzzy membership function diagram of unit space, as shown in Fig2.

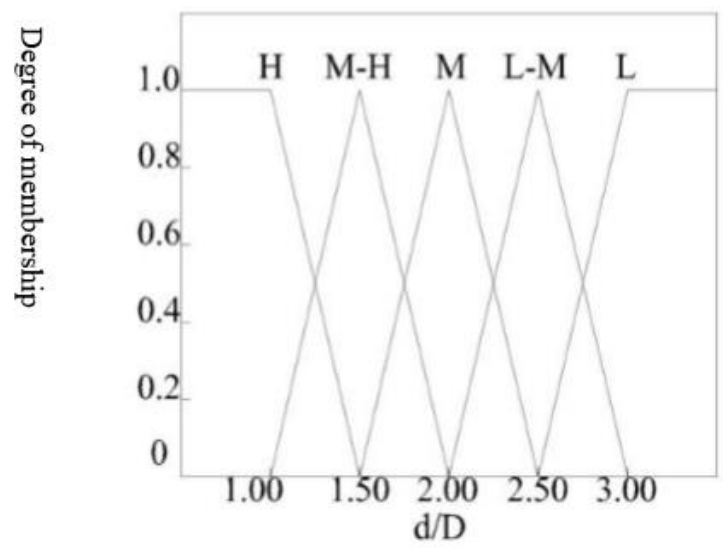

Fig2 The fuzzy membership function diagram of unit distance

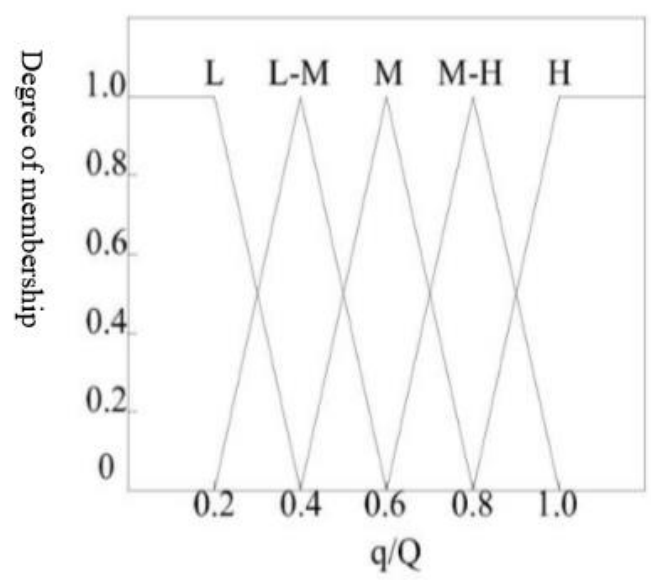

Fig3 Fuzzy membership function diagram of medium inventory

\subsubsection{Fuzzy membership function of medium inventory}

The ratio of the actual quantity of medium $(q)$ in the unit to the critical value $(Q)$ of hazardous chemicals was taken as the fuzzy variable of the fuzzy function, that is $q / Q$. The critical value of hazardous chemicals refers to the minimum critical value stipulated in "Identification of Major Hazardous Sources of Hazardous Chemicals" (GB 18218-2009). It is calculated according to formula (2) when there are more than one hazardous chemical in the unit.

$$
\frac{q}{Q}=\frac{q_{1}}{Q_{1}}+\frac{q_{2}}{Q_{2}}+\cdots+\frac{q_{n}}{Q_{n}}
$$

where: $q_{1}, q_{2}, \ldots, q_{n}-$ The actual amount of each hazardous chemical $(\mathrm{t})$;

$Q_{1}, Q_{2}, \ldots, Q_{n}-$ The corresponding critical amount of hazardous chemicals (t).

The fuzzy membership function of medium inventory can be obtained as shown in Fig. 3. The congestion degree of medium inventory can be divided into five levels of "low (L)", low -medium (L-M)", "medium (M)", "medium-high (M-H)" and "high (H)".

\subsubsection{Fuzzy membership function of medium stock}

For combustible gases, the lower explosion limit of the mixture of combustible gases and air was used as the fuzzy variable of the fuzzy function, as shown in Fig. 4. For flammable liquids, the flash point of flammable liquids was regarded as a fuzzy variable of the fuzzy function, as shown in Fig. 5. The classification criterion of the above two parameters refers to the assortment of fire hazards of combustible substances in "Code for Design and Fire Prevention of Petrochemical Enterprises" (GB 50160-2008).

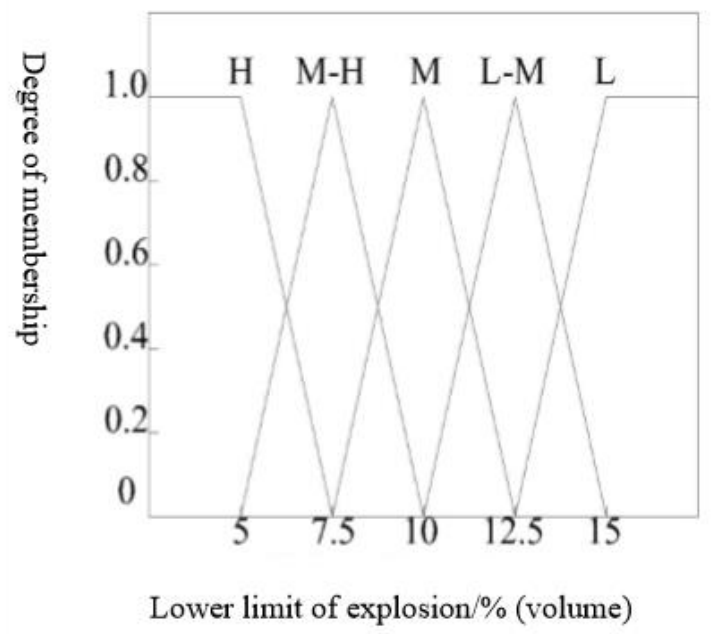

Fig4 Fuzzy membership function diagram of medium hazard (combustible gas) 


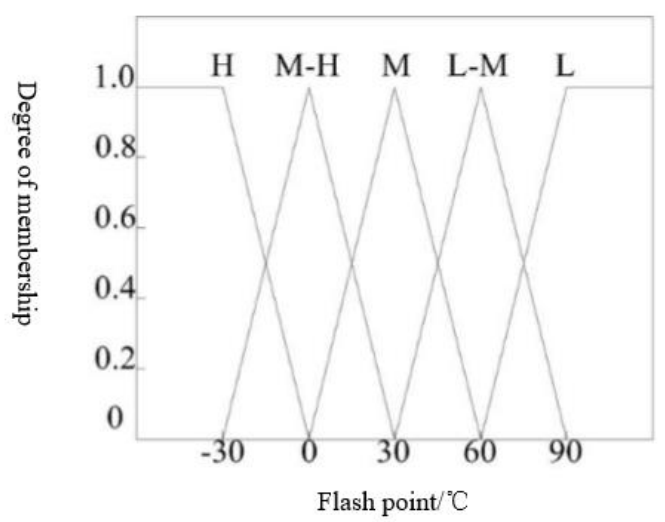

Fig5 Fuzzy membership function diagram of medium hazard (flammable liquid)

\subsubsection{Basic operation rules of fuzzy functions}

The membership degree of each influencing factor can be obtained from Fig. 2 to Fig. 5, and then the fuzzy expression $C D$ of plant layout congestion can be reached in the formula (1). The operation rules used in fuzzy computation are shown in Table 1.

Table1 Rule table of fuzzy function logic operation

\begin{tabular}{|c|c|c|c|}
\hline \multicolumn{3}{|c|}{ Input item } & \multirow{2}{*}{$\begin{array}{l}\text { Output iten } \\
C D\end{array}$} \\
\hline$C D_{s}$ & $C D_{v}$ & $C D_{H}$ & \\
\hline $\mathrm{H}$ & $\mathrm{H}$ & $\mathrm{H}$ & $\mathrm{H}$ \\
\hline $\mathrm{H}$ & $\mathrm{H}$ & M-H & $\mathrm{H}$ \\
\hline $\mathrm{H}$ & $\mathrm{H}$ & M & H \\
\hline $\mathrm{H}$ & $\mathrm{H}$ & L-M & $\mathrm{M}-\mathrm{H}$ \\
\hline $\mathrm{H}$ & $\mathrm{H}$ & L & $\mathrm{M}-\mathrm{H}$ \\
\hline $\mathrm{H}$ & M-H & $\mathrm{M}-\mathrm{H}$ & $\mathrm{M}-\mathrm{H}$ \\
\hline $\mathrm{H}$ & M-H & M & $\mathrm{M}-\mathrm{H}$ \\
\hline $\mathrm{H}$ & M-H & L-M & $\mathrm{M}-\mathrm{H}$ \\
\hline $\mathrm{H}$ & M-H & L & M \\
\hline $\mathrm{H}$ & $\mathrm{M}$ & M & M \\
\hline $\mathrm{H}$ & M & L-M & M \\
\hline $\mathrm{H}$ & M & $\mathrm{L}$ & M \\
\hline $\mathrm{H}$ & L-M & L-M & M \\
\hline $\mathrm{H}$ & L-M & $\mathrm{L}$ & L-M \\
\hline $\mathrm{H}$ & L & L & L-M \\
\hline $\mathrm{M}-\mathrm{H}$ & M-H & $\mathrm{M}-\mathrm{H}$ & $\mathrm{M}-\mathrm{H}$ \\
\hline $\mathrm{M}-\mathrm{H}$ & M-H & M & M \\
\hline M-H & $\mathrm{M} \cdot \mathrm{H}$ & L-M & M \\
\hline $\mathrm{M}-\mathrm{H}$ & $\mathrm{M} \cdot \mathrm{H}$ & L & M \\
\hline M-H & $\mathrm{M}$ & M & M \\
\hline $\mathrm{M}-\mathrm{H}$ & M & L-M & M \\
\hline M-H & M & $\mathrm{L}$ & M \\
\hline M-H & L-M & L-M & L-M \\
\hline M-H & L-M & $\mathrm{L}$ & L-M \\
\hline $\mathrm{M}-\mathrm{H}$ & L & L & L-M \\
\hline M & M & $\mathrm{M}$ & M \\
\hline M & M & L-M & M \\
\hline M & M & L & M \\
\hline M & L-M & L-M & L-M \\
\hline M & L-M & $\mathrm{L}$ & L-M \\
\hline M & L & L & L-M \\
\hline L-M & L-M & L-M & L-M \\
\hline L-M & L-M & L & L-M \\
\hline L-M & L & L & L \\
\hline $\mathrm{L}$ & L & L & $\mathrm{L}$ \\
\hline
\end{tabular}

\subsection{Risk analysis of domino accident}

The risks brought by domino accidents to human beings can generally be divided into two categories: individual risk (IR) and social risk (SR) in the domino effect risk analysis of petrochemical plant accidents.

(1) Individual risk

The definition of personal risk value generated by domino effect in a certain area is shown in Formula (5) in the domino accident.

$$
\mathrm{IR}=\sum_{i=1}^{n} f_{\mathrm{d}}^{(k, m)} \mathrm{V}_{d}^{(k, m)}
$$

where: $I R$ is the individual risk caused by domino effect somewhere; $f_{\mathrm{d}}^{(k, m)}$ is frequency of accidents; $\mathrm{V}_{d}^{(k, m)}$ is mortality.

The calculating steps of the accident mortality can be according to formulas (6) and (7).

$$
\begin{gathered}
V=\frac{1}{\sqrt{2 \pi}} \int_{-\infty}^{Y-5} e^{-\frac{u^{2}}{2}} d u \\
Y=a+b \ln D
\end{gathered}
$$

where: $Y$ is the value of the damage variable; $D$ is dose; $a, b$ all is parameter.

The parameters of human vulnerability model affected by different domino factors are shown in Table 2.

Table2 Human vulnerability models under different domino influence factors

\begin{tabular}{cccc}
\hline Influence factor & $\mathrm{a}$ & $\mathrm{b}$ & $\mathrm{D}$ \\
\hline Thermal radiation & -37.23 & 2.56 & $D=I^{1.33} t$ \\
Overpressure & 5.13 & 1.37 & $D=P_{S}$ \\
Leak $\left(\mathrm{N}_{2}\right)$ & -9.82 & 0.71 & $D=C^{2} t$ \\
Leak $\left(\mathrm{Cl}_{2}\right)$ & -10.1 & 1.11 & $D=C^{1.65} t$ \\
\hline
\end{tabular}

where: $I$ is thermal radiation intensity $\left(\mathrm{W} / \mathrm{m}^{2}\right) ; t$ is the time that acts on the human body(s); $C$ is concentration of leaked poisons (ppm).

(2) Social risk

The social risk value of domino accident in a certain area can be calculated by Formula (8).

$$
S R=\rho \times R
$$

where: $\rho$ is the population density in the region.

\subsection{Study on influence of congestion degree of petrochemical plant layout on domino effect}

In the related research of domino effect in petrochemical plant area, domino effect was mainly divided into domino probability analysis and domino risk analysis. The mechanism of domino effect caused by layout congestion of petrochemical plant area will be qualitatively analyzed from the above two aspects.

\subsubsection{Effect of plant layout congestion degree on occurrence and damage probability of domino effect}

(1) Equipment damage probability

In the calculation and analysis of the equipment damage probability model studied by previous scholars (Cozzani, Gubinelli \& Antonioni, 2005; Salzanoa and Cozzani, 2006; Cozzani, Antonioni \& Landucci, 2014; Gubinelli \& Zanelli, 2004, 2008, 2009), the probability analysis was carried out separately according to the three physical effects (overpressure, thermal radiation and debris) formed by the initial accident of the domino accident.

In the case of overpressure and heat radiation, the damage probability of the equipment can be calculated 
and analyzed by

$$
\mathrm{P}=\frac{1}{\sqrt{2 \pi}} \int_{-\infty}^{Y-5} e^{-\frac{x^{2}}{2}} d x
$$

and the damage variable $Y$. For the case of overpressure, the probability of equipment damage was mainly related to the peak value of static overpressure, which was less affected by the congestion degree of plant layout defined in this paper. In the case of thermal radiation, the damage variable of the equipment varies with the volume of the unit and the intensity of the target unit affected by the thermal radiation of the adjacent unit. In the case of thermal radiation, the damage variable of the equipment changes with the volume of the unit and the radiation intensity of the target unit by adjacent unit. To some extent, the volume of equipment unit reflects the amount of medium stock that has an impact on congestion degree, while the thermal radiation intensity of target equipment unit increases with the decrease of unit spacing. Therefore, the smaller the unit spacing, the larger the medium inventory and the larger the layout congestion will cause the greater the probability of equipment damage under thermal radiation.

In the case of debris, the probability of equipment damage is affected by debris penetration, impact and collision. The probability of debris penetration and collision is influenced by the distance between devices, and the reduction of it will be reflected in the increase of congestion. The impact energy of debris is related to the thermal radiation of the equipment, which is the same as the thermal radiation mentioned above.

(2) Probability of domino scene

The increase of intraregional the degree of congestion means that the cell spacing decreases, which leads to the increase of the number of units in the region. As a result, the number of secondary events caused by an initial event will rise sharply, and the impact of the domino effect will expand, eventually increasing the probability of domino scenarios.

\subsubsection{The impact of factory layout congestion degree on the risk of domino effect}

(1) Risk analysis of domino consequences based on accidents

\section{(1) Leakage accident}

The extent of damage from the leakage of hazardous substances is proportional to the amount of leakage of the substance. A liquid pool will be formed after the liquid leaks out of the target equipment unit whose leakage from evaporation is related to the area and quality of the liquid pool formed. Therefore, it can be seen that if the inventory amount in the target unit device is larger, the layout congestion degree is increased. And the area of the pool formed by the leak and the quality of the leaked liquid are also greater. Thus, the greater the amount of leakage is, the greater the radius of death for the target equipment unit to leak is.

(2) Fire and explosion accident

The damage to the human body is mainly caused by thermal radiation and overpressure in fire and explosion accidents. The relationship between layout congestion and thermal radiation and overpressure was referred to in Section 2.3.1.

(2) Individual risk and social risk

The personal risk value is determined by the sum of the product of the accident frequency and the accident mortality rate of the adjacent equipment units. Therefore, it may increase the frequency of accidents and mortality caused by accidents in the area when the layout congestion of a certain location is large (possibly smaller inter-unit spacing, larger unit inventory or greater risk of medium in the unit). As a result, the individual risk of the area rises.

Social risk is a risk evaluation index on account of population density in the region which the social risk in a certain region increases with the augment of the distribution congestion under the same population density.

\section{Case study and results analysis}

The tank area of an aniline plant and a refinery in a chemical park was selected as a case study. The location of specific units was shown in Fig. 6. The properties of medium were shown in Table 3.

Table3 Properties of medium in tank area

\begin{tabular}{|c|c|c|c|c|c|c|c|}
\hline $\begin{array}{l}\text { Device } \\
\text { number }\end{array}$ & $\begin{array}{c}\text { Owned } \\
\text { enterprise }\end{array}$ & $\begin{array}{c}\text { Medium } \\
\text { name }\end{array}$ & $\begin{array}{c}\text { Volume of } \\
\text { single } \\
\text { storage tank } \\
\left(\mathrm{m}^{3}\right)\end{array}$ & $\begin{array}{c}\text { Storage } \\
\text { temperature } \\
\left({ }^{\circ} \mathrm{C}\right)\end{array}$ & $\begin{array}{l}\text { Design } \\
\text { pressure } \\
(\mathrm{Pa})\end{array}$ & Risk & $\begin{array}{r}\text { Inventory } \\
\text { of a single } \\
\text { tank (kg) }\end{array}$ \\
\hline $\begin{array}{l}\text { v101- } \\
\text { v103 }\end{array}$ & Aniline plant & Benzene & 3000 & $\begin{array}{c}\text { Ordinary } \\
\text { temperature }\end{array}$ & 104325 & $\begin{array}{l}\text { Major hazard } \\
\text { sources }\end{array}$ & 65000 \\
\hline v104 & Aniline plant & Aniline & 300 & $\begin{array}{c}\text { Ordinary } \\
\text { temperature }\end{array}$ & 101325 & $\begin{array}{l}\text { Non major hazard } \\
\text { sources }\end{array}$ & 20000 \\
\hline V105 & Aniline plant & Nitrobenzene & 250 & $\begin{array}{c}\text { Ordinary } \\
\text { temperature }\end{array}$ & 101325 & $\begin{array}{c}\text { Non major hazard } \\
\text { sources }\end{array}$ & 25000 \\
\hline V106 & Aniline plant & Naphtha & 200 & $\begin{array}{l}\text { Ordinary } \\
\text { temperature }\end{array}$ & 101325 & $\begin{array}{l}\text { Non major hazard } \\
\text { sources }\end{array}$ & 9000 \\
\hline V107 & Aniline plant & $\begin{array}{l}\text { Nitric acid } \\
(98 \%)\end{array}$ & 300 & $\begin{array}{c}\text { Ordinary } \\
\text { temperature }\end{array}$ & 101325 & $\begin{array}{c}\text { Non major hazard } \\
\text { sources }\end{array}$ & 35000 \\
\hline V108 & Aniline plant & $\begin{array}{c}\text { Sulphuric } \\
\text { acid (98\%) }\end{array}$ & 100 & $\begin{array}{c}\text { Ordinary } \\
\text { temperature }\end{array}$ & 101325 & $\begin{array}{l}\text { Major hazard } \\
\text { sources }\end{array}$ & 15000 \\
\hline $\mathrm{C} 101$ & Aniline plant & Benzene & 2000 & $\begin{array}{c}\text { Ordinary } \\
\text { temperature }\end{array}$ & 104325 & $\begin{array}{l}\text { Major hazard } \\
\text { sources }\end{array}$ & 35000 \\
\hline $\begin{array}{l}\text { V201- } \\
\text { V206 }\end{array}$ & Refinery & Gasoline & 5000 & $\begin{array}{c}\text { Ordinary } \\
\text { temperature }\end{array}$ & 101325 & $\begin{array}{l}\text { Major hazard } \\
\text { sources }\end{array}$ & 75000 \\
\hline $\begin{array}{l}\text { v207. } \\
\text { v210 }\end{array}$ & Refinery & Kerosene & 2000 & $\begin{array}{c}\text { Ordinary } \\
\text { temperature }\end{array}$ & 101325 & $\begin{array}{l}\text { Major hazard } \\
\text { sources }\end{array}$ & 15000 \\
\hline
\end{tabular}

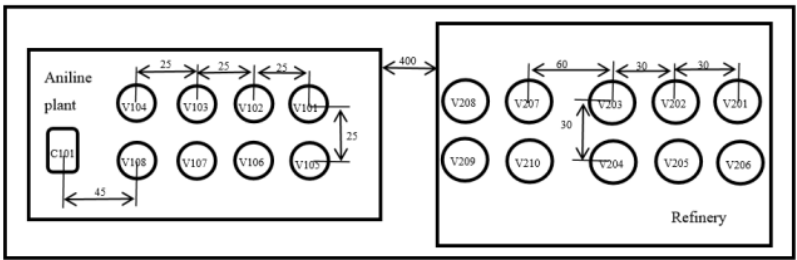

Fig6 Layout (part) of chemical industry park.

\subsection{The effect of layout congestion on domino risk}

In order to study the effect mechanism of layout congestion on the risk of domino effect, V102, V104, C101, V106 and V107 storage tanks in the factory were selected. The calculation of the congestion degree of the layout was shown in Table 4. 
Table4 Layout congestion degree of storage tanks

Layout congestion degree: V102 $>$ V106 $>$ C101 $>$ V104 $>$ V107

\begin{tabular}{ccccc}
\hline Unit number & $C D_{s}$ & $C D_{\nu}$ & $C D_{H}$ & $C D$ \\
\hline v102 & $\frac{0.60}{M-H}+\frac{0.40}{M}$ & $\frac{1.00}{H}$ & $\frac{0.37}{H}+\frac{0.63}{M-H}$ & $\frac{0.37}{H}+\frac{0.40}{M-H}$ \\
v104 & $\frac{0.40}{M}+\frac{0.60}{L-M}$ & $\frac{1.00}{L}$ & $\frac{0.67}{L-M}+\frac{0.33}{L}$ & $\frac{0.33}{L-M}+\frac{0.33}{L}$ \\
C101 & $\frac{1.00}{L}$ & $\frac{1.00}{H}$ & $\frac{0.37}{H}+\frac{0.63}{M-H}$ & $\frac{0.37}{M-H}+\frac{0.63}{M}$ \\
v106 & $\frac{0.20}{M}+\frac{0.80}{L-M}$ & $\frac{0.50}{H}+\frac{0.50}{M-H}$ & $\frac{0.13}{H}+\frac{0.87}{M-H}$ & $\frac{0.13}{H}+\frac{0.13}{M-H}+\frac{0.20}{M}$ \\
v107 & $\frac{0.40}{M}+\frac{0.60}{L-M}$ & $\frac{0.75}{L-M}+\frac{0.25}{L}$ & $\frac{1.00}{L}$ & $\frac{0.25}{L-M}+\frac{0.25}{L}$ \\
\hline
\end{tabular}

The individual risk values of dominoes for each tank were shown in Table 5 9. The equipment failure probability of each tank is $1.5 \times 10^{-5} / \mathrm{a}^{-1}$, the density of population of person $/ \mathrm{m}^{2}$.

Table5 Risk value of V102 tank.

\begin{tabular}{cccc}
\hline Distance from V102 & Mortality & IR & SR \\
\hline 25 & 1.000 & $1.500 \times 10^{-5}$ & $1.500 \times 10^{-7}$ \\
50 & 0.811 & $1.217 \times 10^{-5}$ & $1.217 \times 10^{-7}$ \\
75 & 0.605 & $9.075 \times 0^{-6}$ & $9.075 \times 0^{-8}$ \\
100 & 0.223 & $3.345 \times 10^{-6}$ & $3.345 \times 10^{-8}$ \\
\hline
\end{tabular}

Table6 Risk value of V104 tank.

\begin{tabular}{|c|c|c|c|}
\hline Distance from V104 & Mortality & IR & SR \\
\hline 25 & 0.683 & $1.025 \times 10^{-5}$ & $1.025 \times 10^{-7}$ \\
\hline 50 & 0.532 & $7.980 \times 10^{-6}$ & $7.980 \times 10^{-8}$ \\
\hline 75 & 0.356 & $5.340 \times 10^{-6}$ & $5.340 \times 10^{-8}$ \\
\hline 100 & 0.098 & $1.470 \times 10^{-6}$ & $1.470 \times 10^{-8}$ \\
\hline \multicolumn{4}{|c|}{ Table7 Risk value of C101 tank. } \\
\hline Distance from $\mathrm{C} 101$ & Mortality & IR & SR \\
\hline 25 & 1.000 & $1.500 \times 10^{-5}$ & $1.500 \times 10^{-7}$ \\
\hline 50 & 0.802 & $1.203 \times 10^{-5}$ & $1.203 \times 10^{-7}$ \\
\hline 75 & 0.592 & $8.880 \times 10^{-6}$ & $8.880 \times 10^{-8}$ \\
\hline 100 & 0.203 & $3.045 \times 10^{-6}$ & $3.045 \times 10^{-8}$ \\
\hline \multicolumn{4}{|c|}{ Table8 Risk value of V106 tank. } \\
\hline Distance from V106 & Mortality & IR & SR \\
\hline 25 & 1.000 & $1.500 \times 10^{-5}$ & $1.500 \times 10^{-7}$ \\
\hline 50 & 0.807 & $1.211 \times 10^{-5}$ & $9.733 \times 10^{-7}$ \\
\hline 75 & 0.601 & $9.015 \times 10^{-6}$ & $9.015 \times 10^{-8}$ \\
\hline 100 & 0.218 & $3.270 \times 10^{-6}$ & $3.270 \times 10^{-s}$ \\
\hline \multicolumn{4}{|c|}{ Table9 Risk value of V107 tank. } \\
\hline Distance from V107 & Mortality & IR & SR \\
\hline 25 & 0.633 & $9.500 \times 10^{-5}$ & $9.500 \times 10^{-8}$ \\
\hline 50 & 0.501 & $7.515 \times 10^{-6}$ & $7.515 \times 10^{-8}$ \\
\hline 75 & 0.300 & $4.500 \times 10^{-5}$ & $4.500 \times 10^{-8}$ \\
\hline 100 & 0.061 & $9.150 \times 10^{-7}$ & $9.150 \times 10^{-9}$ \\
\hline
\end{tabular}

Fig7 (Individual risk diagram of tanks with distance) and Fig8 (The change of individual risk with congestion degree at different distances) can be drawn from the individual risk values of domino produced by the tanks at different distances in Tables 5 to 9.

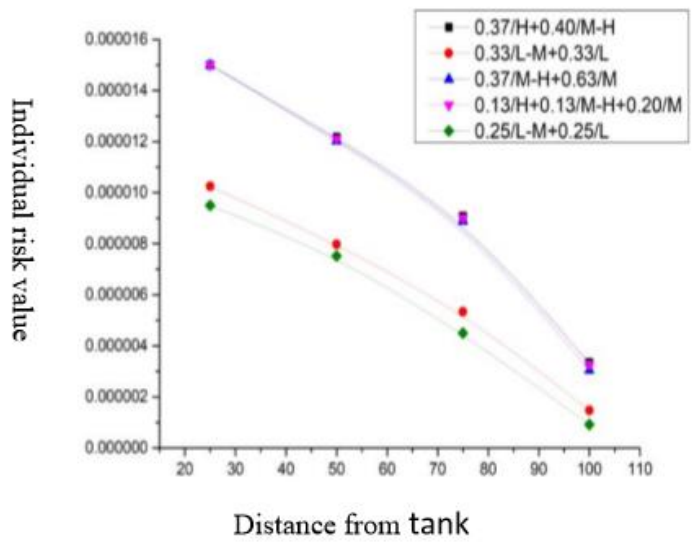

Fig7 Distance-individual risk value

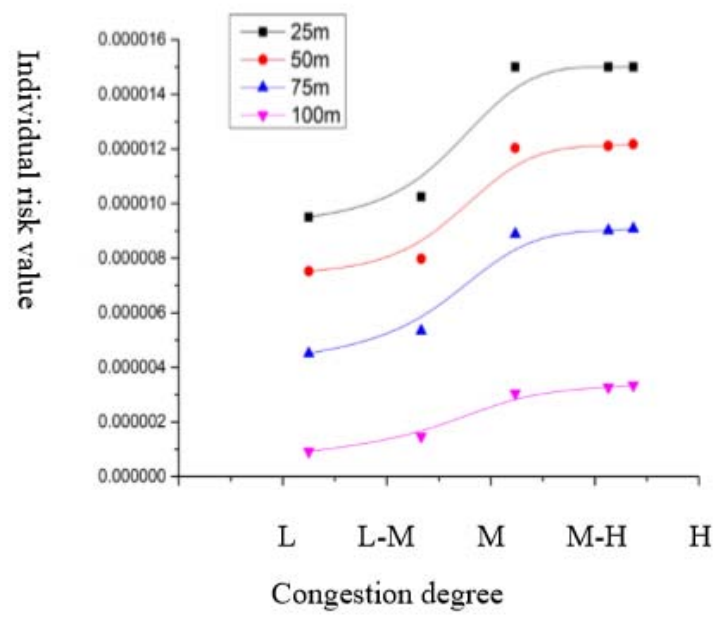

Fig8 Congestion degree - individual risk value

According to Fig. 7, the change trend of individual risk value decreased with the increase of distance from the unit, and the greater the individual risk value associated with the greater the congestion degree at the unit. Fig. 8 shows that the individual risk value of the unit increases with congestion degree at the unit. Moreover, the increase rate of personal risk value is from rising gently to a sharp rise and eventually to a mild level. The maximum growth rate of the individual risk value obtained by the four curve of the graph is at the point where the congestion degree is $\frac{0.81}{M}$.

In this case, congestion degree can be divided into four areas according to individual risk value, as shown in Table 10.

Table10 Hierarchy of layout congestion degree

\begin{tabular}{|c|c|c|c|}
\hline The range in Fig. 4 & Congestion degree range & Danger grade & Degree of recommendation \\
\hline $0 \sim[1]$ & $0 \sim \frac{0.41}{L-M}+\frac{0.38}{L}$ & Safe & \multirow{2}{*}{ Recommend } \\
\hline$[1\}-[2]$ & $\frac{0.41}{L-M}+\frac{0.38}{L} \sim \frac{0.83}{L-M}$ & Little safe & \\
\hline$[2\}-[3]$ & $\frac{0.83}{L-M} \sim \frac{0.78}{M}$ & Little unsafe & Not recommended \\
\hline$>[3]$ & $\frac{0.78}{M} \sim \frac{1.00}{H}$ & Danger & Suggest avoiding \\
\hline
\end{tabular}

In Table $10,{ }^{[1]}$ is corresponding to degree of congestion of $\frac{0.41}{L-M}+\frac{0.38}{L}$. When the congestion degree is less than this value, the individual risk value of the facility has been below $1 \times 10^{-6}$ within $100 \mathrm{~m}$ of the target unit facility, and the domino risk of unit equipment was two orders of magnitude lower than the failure rate of the equipment, and the risk of domino was controlled. The degree of congestion of ${ }^{[2]}$ is $\frac{0.83}{L-M}$. At this congestion level, the individual risk value corresponding to $25 \mathrm{~m}$ from the unit facility is $1 \times 10^{-5}$ and the failure rate of the equipment is $1 \times 10^{-5} / \mathrm{a}^{-1}$. It can be seen that the risk of domino at the nearer distance from the target unit is one order of magnitude lower than the failure rate of the equipment within this range, and the risk of domino is more controllable as well. [3] corresponded to a congestion value of $\frac{0.78}{M}$, where the individual risk value of domino generated at $50 \mathrm{~m}$ from the unit facility was equal to $1 \times 10^{-5}$. At this point, the consequences will be more difficult to control due to the larger scope and 
intensity of the domino effect. Therefore, the four scope of layout congestion degree was shown in Table 10.

According to the results of the research, the layout congestion degree should be within the recommended range as far as possible in the layout of the plant area. However, the existence of major hazards was inevitable under certain conditions due to the material and process characteristics in the plant area. It can be known from the definition of layout congestion that when the risk of materials and the congestion of inventory are large, the purpose of reducing layout congestion degree and domino risk can be achieved by increasing the safe distance between units.

\section{Conclusion}

The purpose is that to study the effect of layout congestion on the risk of domino effect in petrochemical plants. The main results were as follows.

Firstly, based on fuzzy mathematics and the principle of maximum membership degree, a fuzzy model of congestion degree in petrochemical plant area was established, which made the congestion degree of a certain place in petrochemical plant area can be expressed by the membership function of congestion degree, and made simultaneously the congestion degree at different locations comparable quantitatively.

Secondly, it was concluded that the risk of domino effect amplified with the enlargement of congestion degree in view of the consequence analysis value of accident cause, individual risk and social risk.

Thirdly, five storage tanks in aniline plant were selected for quantitative risk analysis. Individual risk values were used as indicators to characterize domino risk. The change of individual risk values with layout congestion degree was plotted according to the results. Finally, the recommended layout congestion degree range which has a certain guiding role in plant layout was obtained by dividing the risk area range.

\section{Acknowledgments}

The financial support of the Natural Science Foundation of Shanghai (16ZR1408500), the Technology Development Foundation of China Sinopec Qingdao Safety Engineering Institute (313038), the project for Development of Systems of Quantitative Risk Assessment of Domino Effect in the Chemical Industry (B100-81414), the Natural Science Foundation of Jiangsu Province (BK2012824) and Innovation Practice Training Program for college students (Accident risk analysis of typical ground and underground installations in petrochemical industry, S19098) are gratefully acknowledged.

\section{References}

1. Alileche, N., Cozzani, V., Reniers, G.(2015) Thresholds for domino effects and safety distances in the process industry: A review of approaches andregulations. Reliability Engineering\&System Safety., 143: 74-84.

2. Antonioni, G., Spadoni, G., Cozzani, V. (2009) Application of domino effect quantitative risk assessment to an extended industrial area. Journal of Loss Prevention in the Process Industries., 22(5): 614-624.

3. Abdolhamidzadeh, B., Abbasi, T., Rashtchian, D.(2010) A new method for assessing domino effect in chemical process industry. Journal of Hazardous Materials., 182(1-3): 416-426. 